Voix et Images

voixetimages

\title{
Interdisciplinarités
}

\section{Robert Dion}

Volume 35, numéro 3 (105), printemps-été 2010

Les mémorialistes québécois du XIX ${ }^{\mathrm{e}}$ siècle

URI : https://id.erudit.org/iderudit/044266ar

DOI : https://doi.org/10.7202/044266ar

Aller au sommaire du numéro

Éditeur(s)

Université du Québec à Montréal

ISSN

0318-9201 (imprimé)

1705-933X (numérique)

Découvrir la revue

Citer ce compte rendu

Dion, R. (2010). Compte rendu de [Interdisciplinarités]. Voix et Images, 35(3), 135-139. https://doi.org/10.7202/044266ar

Ce document est protégé par la loi sur le droit d'auteur. L'utilisation des services d'Érudit (y compris la reproduction) est assujettie à sa politique d'utilisation que vous pouvez consulter en ligne.

https://apropos.erudit.org/fr/usagers/politique-dutilisation/ 


\title{
E S S A I S / ÉT U D E S
}

Interdisciplinarités

\author{
$++$ \\ ROBERT DION \\ Université du Québec à Montréal
}

Un grand plaisir que m'aura procuré cette chronique sans doute un peu trop irrégulièrement tenue - et dont c'est aujourd'hui la dernière mouture - aura été celui de prendre connaissance du renouveau des travaux d'histoire et d'érudition au Québec. La lecture commandée des dernières recherches dans ce domaine m'aura ainsi conduit à faire ce qu'on ne peut, hélas! se permettre si souvent: lire d'un bout à l'autre, sans en sauter une seule ligne, des sommes telles que La vie littéraire au Québec ou encore Histoire de la littérature québécoise de Michel Biron, François Dumont et Élisabeth Nardout-Lafarge ${ }^{1}$. Grâce à de telles lectures, je serai parvenu à consolider certaines connaissances jusque-là chambranlantes, à colmater des brèches et des lacunes abyssales, à me constituer aussi, j'espère, un certain savoir positif.

L'attention soutenue que j'ai accordée à plusieurs collectifs importants m'a également permis d'observer une certaine dissolution de l'objet littéraire qui n'aurait pas été imaginable, je crois, à l'ère du structuralisme et de la sémiotique triomphants, qui avaient justement cherché à construire l'objet et à établir sa spécificité. Je n'irai pas jusqu'à déplorer cet état de fait - ce qui reviendrait, peu ou prou, à me «dater"; force m'est toutefois de relever que la littérature, dans beaucoup de travaux d'érudition actuels sur l'histoire de la culture québécoise, a souvent droit à la part congrue. Est-ce le signe d'une perte d'interêt de la part du lectorat savant? L'indice d'une certaine saturation de l'objet? L'indication d'un déplacement vers des corpus plus nouveaux et, partant, plus attirants?

Le somptueux premier numéro de la Revue de Bibliothèque et Archives nationales du Québec ${ }^{2}$ est un exemple éloquent de ce rétrécissement de l'espace consacré aux

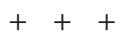

1 C'est l'un de mes cuisants regrets de n'avoir pu parler de cet ouvrage à Voix et Images et de certains autres qui m'ont beaucoup intéressé, mais dont les auteurs sont des amis ou des collègues proches, trop proches. Étroitesse du milieu des études québécoises, parfois... 2 Revue de Bibliothèque et Archives nationales du Québec, $\mathrm{n}^{\circ} 1,2009,123 \mathrm{p}$. 
études littéraires. Ici, rien, ou presque, sur les archives d'écrivains: un simple petit topo de Mariloue Sainte-Marie sur le Fonds Paul-Marie Lapointe, avec beaucoup d'illustrations (bien choisies et bien mises en page, là n'est pas la question) et peu de texte. Était-ce là tout ce qu'il y avait à glaner dans les archives nationales? Bien évidemment, non. N'y a-t-il donc eu aucune proposition valable, à l'interne ou à l'externe, sur les archives d'écrivains, les manuscrits conservés, les entreprises d'édition critique? J'ose espérer que ce n'est pas le cas. Il reste que j'ai été surpris, et decçu, par cette absence, surtout qu'il s'agit d'une première livraison qui donnera le ton à la suite; je l'ai été d'autant plus que le mandat ${ }^{3}$ et la facture de la revue m'apparaissent tout à fait propices à la publication de ce type de travaux. Ce n'est pas si souvent que les périodiques consacrés à l'étude des fonds littéraires peuvent bénéficier d'une présentation aussi soignée et à ce point luxueuse, avec de multiples reproductions et illustrations en couleurs sur des pages grand format. Ce n'est que partie remise, souhaitons-le...

Une fois revenu de ma déception, j'ai dû reconnaître que cette première livraison est excellente. On est loin, très loin ici de la publication «marketing» où le déploiement de moyens tient lieu de contenu éditorial. Les articles sont sérieux, voire carrément austères en certaines occasions (l'histoire de la constitution de la bibliothèque du Séminaire de Rimouski et celle des archives judiciaires dans le Québec britannique tout spécialement). D'autres se caractérisent cependant par un effort d'ouverture à un lectorat moins ciblé; j'en veux pour preuve, entre autres, une étude passionnante sur la cartographie de la Nouvelle-France au XviII siècle dans laquelle Jean-François Palomino décrit la position délicate de cartographes des Lumières coincés entre leurs propres exigences scientifiques et les intérêts politiques de leur commanditaire. Deux articles sur l'histoire de l'urbanisme ont également retenu mon attention, l'un sur la reconstruction du Faubourg Québec après l'incendie du 10 juillet 1852, qui prend appui aussi bien sur le cadastre que sur les actes notariés et sur les esquisses d'architectes, et qui montre comment les déterminations culturelles se trouvent au principe de la reconstruction, l'autre sur le Foyer coopératif de Chicoutimi, exemple significatif de cité-jardin conçue dans les années 1940 et 1950 par des gens désireux d'expérimenter une nouvelle façon de vivre et de construire. Me frappent particulièrement, dans ces recherches, la diversité et la richesse des archives mises à contribution, de même que la capacité des auteurs à faire valoir les multiples facettes des questions envisagées.

Par ailleurs, ceux et celles qui s'intéressent à l'histoire du spectacle ne manqueront pas de consulter l'étude de ce que Danielle Léger et Isabelle Robitaille appellent les ephemera spectaculi, qui comprennent les affiches et programmes du XIX siècle de la Collection patrimoniale de BAnQ; même si l'échantillon est relativement réduit, il y a là de bonnes indications sur le contexte culturel du divertissement

3 À la rubrique «À propos de la revue», l'on précise que celle-ci «accueille en ses pages des articles d'érudition qui s'inscrivent dans l'un ou l'autre des deux créneaux privilégiés par l'institution, soit des recherches exploitant des corpus tirés des fonds et des collections patrimoniales de Bibliothèque et Archives nationales du Québec ou des études portant sur des sujets liês aux missions de l'institution", p. 1. 
de l'époque, qui pourront nourrir de futurs travaux sur la vie culturelle des siècles passés à Montréal.

Autre publication multidisciplinaire et parfois (quoique plus rarement) interdisciplinaire, le livre publié sous la direction d'Yvan Lamonde et Denis Saint-Jacques, 1937 : un tournant culturel ${ }^{4}$, s'inscrit dans le sillage de recherches globales sur l'histoire culturelle du Québec et constitue, après l'ouvrage sur La vie culturelle à Montréal vers $1900^{5}$, un autre jalon de l'effort collectif pour élaborer un récit intégré des pratiques culturelles au siècle dernier. Disons d'abord que là où le livre publié sous la direction de Micheline Cambron se caractérisait par une présentation léchée et par une édition soignée, celui que viennent de lancer les Presses de l'Université Laval a quelque chose de brouillon qui agace un tantinet: qu'il s'agisse des fautes ou des coquilles, des allusions parfois gommées, parfois non, à l'origine orale des textes publiés (un colloque tenu en 2007), de l'absence de références directes aux illustrations à l'intérieur de certains articles, voire du maintien de l'énonciation à la première personne du singulier dans un texte cosigné par deux auteurs, il y a dans ce livre plusieurs petites négligences qu'un peu de soin aurait permis de faire disparaître. Et c'est sans compter que certains articles auraient demandé à être étoffés ou refondus complètement. En lisant ce livre, je me suis étonné de constater, encore une fois, que des auteurs arrivent en une quinzaine de pages à boucler un propos riche, complet et informatif, alors que d'autres n'offrent que de rares aperçus et quelques promesses.

Et l'on ne me fera pas dire que c'est mon parti pris littéraire qui m'amène à préférer les textes bien construits et bien écrits à ceux qui se contentent d'aller à l'essentiel. Quoique leur travail se veuille efficace et factuel, j'ai eu du mal à saisir, par exemple, où voulaient en venir Gérard Fabre dans son étude sur André Siegfried et les milieux canadiens ou Philippe Dubé avec sa thèse - mystérieuse à souhait selon laquelle plus François Hertel collectionne les œuvres d'art et moins son travail philosophique est pertinent. Quant à l'étude de Vincent Charles Lambert sur SaintDenys Garneau, malgré la qualité de la rédaction et le souci littéraire qui la gouverne, j'y ai surtout discerné une énième tentative de conjecturer sur la crise spirituelle qu'a expérimentée le poète à la suite de la publication de Regards et jeux dans l'espace. Où est, ici, le regard du jeune chercheur?

Quelques articles prometteurs souffrent visiblement du format imposé par le colloque ou par le collectif. Ainsi, l'étude d'Yvan G. Lepage sur les "pays» de Menaud cesse plutôt qu'elle ne se conclut; l'article de Serge Lacasse et Chantal Savoie, qui s'inaugure par un vaste panorama de la chanson populaire en 1937, se

4 Yvan Lamonde et Denis Saint-Jacques (dir.), 1937 : un tournant culturel, Québec, Presses de l'Université Laval, coll. «Cultures québécoises», 2009, 371 p. 5 Micheline Cambron (dir.), La vie culturelle à Montréal vers 1900, Montréal, Fides/Bibliothèque nationale du Québec, 2005, 412 p., 1 CD audio. Au sujet de ce livre, voir ma chronique publiée dans le numéro 92 (vol. XXXI, n² 2, hiver 2006) de Voix et Images. 
réduit finalement à l'étude succincte de deux chansons enregistrées par Ludovic Huot; la chose n'est pas condamnable en soi, mais je vois difficilement comment ces analyses pointues peuvent nous conduire à prendre le pouls de l'an 1937.

Malgré mes réserves, j'ai bien sûr trouvé dans cet ouvrage de quoi satisfaire ma curiosité. L'étude de Karim Larose sur le Deuxième Congrès de la langue française au Canada pointe avec beaucoup d'à-propos une transformation en voie de s'accomplir, par les marges, lors de l'événement: celle qui fait passer d'une injonction de propagande de ce qu'on nomme alors l'«esprit français» à la sommation d'élaborer une véritable culture canadienne-française globale (22-23). Envisagés ensemble, les articles d'Andrée Lévesque sur les midinettes de 1937 et de Robert Schwartzwald sur les rapports entre la communauté juive et les communautés anglo- et francomontréalaises montrent comment les considérations socio-ethniques, notamment, jouent tant sur le plan local et nord-américain - les grèves dans l'industrie du vêtement - que sur celui des relations internationales - l'attitude vis-à-vis de Franco, de Hitler et des propagateurs de leurs idées au Québec. J'ai aussi trouvé un grand profit à lire en parallèle les textes d'Esther Trépanier et de Laurier Lacroix. Les deux historiens de l'art interrogent en effet deux versants de la même modernité: tandis que Trépanier essaie de voir pourquoi les peintres modernistes de 1937, Adrien Hébert puis les artistes juifs de Montréal, ratent leur rendez-vous avec la culture de masse naissante, Lacroix montre, prolongeant par là une idée lancée par sa collègue, que la modernité picturale est plutôt fécondée au contact de l'art populaire, naï et «barbare». Les deux articles, même s'ils ne parlent pas exactement des mêmes artistes, dessinent néanmoins des panoramas absolument compatibles.

Quant au texte de Lucie Robert, c'est davantage dans la foulée de son apport à l'ouvrage dirigé par Micheline Cambron qu'il faut le lire ${ }^{6}$. Alors qu'elle évoquait là une vie théâtrale se réorganisant, autour de 1900, dans un tohu-bohu où il était difficile de dégager quelque orientation claire, Robert dresse ici le portrait d'une situation tout autre à la fin des années 1930 : une époque où, en raison de la crise de 1929 et de l'essor du cinéma, le milieu thêâtral s'est à peu près complètement effondré et où, pourtant, apparaissent quelques lueurs de renouveau avec les figures, encore marginales, du père Émile Legault et de Gratien Gélinas, ainsi qu'avec la fondation du Mont-Royal Thêâtre Français. Karine Cellard s'attache aussi à quelque chose qui se termine et qui recommence autour de 1937: à partir de l'analyse d'un objet circonscrit et singulier, les Pamphlets de Valdombre, elle saisit l'histoire de la critique à un moment où celle-ci, avec la défection de l'éditeur Albert Lévesque, connaît un relatif déclin; à un moment, de même, où le régionalisme intransigeant incarné par Claude-Henri Grignon s'essouffle alors que, par ailleurs, l'esthétique régionaliste, en puisant à des courants plus modernes, connaît un certain regain (Ringuet, Guèvremont, DesRochers, Savard) dans l'exacte mesure où elle défait la bipolarité héritée de la querelle des terroiristes et des exotiques.

Il se trouve donc beaucoup de bon, et de très bon, dans 1937 : un tournant culturel. Bien d'autres travaux consignés dans ce volume auraient mérité d'être cités

$$
+++
$$

6 Lucie Robert, «Chronique de la vie théâtrale», Micheline Cambron (dir.), ouvr. cité, p. 71-86. 
et évoqués; le sommaire de toutes ces idées et analyses se révèle toutefois au-dessus de mes forces, sans compter qu'il mettrait à rude épreuve la patience de mon lecteur. Je terminerai par conséquent en insistant sur la pertinence de la coupe synchronique envisagée pour le colloque et pour le livre: 1937, on s'en convainc au fil de la lecture, c'est effectivement une année charnière, et pour bien d'autres raisons que celle qui nous vient spontanément à l'esprit, soit le centième anniversaire de la rébellion de 1837-1838. L'une des choses que l'on apprend dans ce collectif, c'est que cet événement historique, dans le premier tiers du $\mathrm{Xx}^{\mathrm{e}}$ siècle, est loin d'avoir la résonance qu'il a pour les enfants de la Révolution tranquille que nous sommes. En 1937, Dollard des Ormeaux avait encore de belles décennies devant lui...

En conclusion de l'ouvrage, les éditeurs scientifiques ont eu l'excellente idée de tenter, chacun pour soi, un résumé des avancées du colloque et de l'ouvrage qui en rend compte. Chez Lamonde, les atermoiements du chanoine Groulx au sujet de la question nationale apparaissent particulièrement symptomatiques de la période, comme du reste le malaise de Saint-Denys Garneau devant l'envahissement du religieux par la politique et l'avancée du matérialisme. Pour Saint-Jacques, c'est l'américanisation de la société et de la culture québécoises qui compte vraiment, tout le reste de la donne idéologique se redistribuant en fonction de ce fait «brut» qui, comme il le dit plaisamment, «ne tien[t] aucun congrès, ne publi[e] pas de Directives, n'offr[e] aucun chef-d'œuvre ni coup d'éclat» (366).

Plus que jamais - on le voit aux publications que j'ai abordées dans cette ultime chronique - , les études littéraires avancent de conserve avec d'autres disciplines, la littérature est envisagée avec d'autres arts sans qu'on lui reconnaisse quelque statut particulier. Tout n'est sans doute pas à regretter - ni a fortiori à condamner - dans cette nouvelle, et lourde, tendance de la recherche. Se trouvera-t-il encore quelqu'un, quelque part, pour se désoler qu'on réussisse de plus en plus difficilement, et pour un public de plus en plus restreint, à publier des études monographiques sur un auteur, voire sur une œuvre, sans la renvoyer à tout son "environnement» culturel? Poser la question c'est peut-être, déjā, y répondre. 